\section{Human Heredity}

\author{
Joseph D. Terwilliger ${ }^{\mathrm{a}, \mathrm{b}}$ \\ Sebastian Zöllner ${ }^{\mathrm{C}}$ \\ Maris Laan ${ }^{\mathrm{C}}$ \\ Svante Pääbo \\ a Department of Psychiatry and \\ Columbia Genome Center, \\ Columbia University and \\ ${ }^{b}$ Department of Neuroscience, \\ New York State Psychiatric \\ Institute, New York., N.Y., USA; \\ c Institute of Zoology, University of \\ Munich, Germany
}

\section{Key Words}

Genetic drift

Linkage disequilibrium

Demographic history

Population isolate

Complex disease

Population structure

Saami

Finns

\title{
Mapping Genes through the Use of Linkage Disequilibrium Generated by Genetic Drift: 'Drift Mapping' in Small Populations with No Demographic Expansion
}

\begin{abstract}
Linkage disequilibrium has been a powerful tool in identifying rare disease alleles in human populations. To date, most research has been directed to isolated populations which have undergone a bottleneck followed by rapid exponential expansion. While this strategy works well for rare diseases in which all disease alleles in the population today are clonal copies of some common ancestral allele, for common disease genes with substantial allelic heterogeneity, this approach is not predicted to work. In this paper, we describe the dynamics of linkage disequilibrium in populations which have not undergone a demographic expansion. In these populations, it is shown that genetic drift creates disequilibrium over time, while in expanded populations, the disequilibrium decays with time. We propose that common disease alleles might be more efficiently identified by drift mapping - linkage disequilibrium mapping in small, old populations of constant size where the disequilibrium is the result of genetic drift, not founder effect. Theoretical models, empirical data, and simulated population models are presented as evidence for the utility of this approach.
\end{abstract}

\section{Introduction}

While population geneticists study the dynamics of linkage disequilibrium in populations, genetic epidemiologists and gene map- pers use linkage disequilibrium as a tool to localize genetic factors involved in the etiology of diseases. Here, we outline an intuitive description of the various evolutionary forces which create and dissolve linkage disequilib-

\begin{tabular}{ll}
\hline KARGER & ( 1998 S. Karger AG, Basel \\
Fax +4161306 12 34 & 0001-5652/98/0483-0138\$15.00/0 \\
$\begin{array}{l}\text { E-Mail karger@karger.ch } \\
\text { www.karger.com }\end{array}$ & $\begin{array}{l}\text { This article is also accessible online at: } \\
\text { http://BioMedNet.com/karger }\end{array}$
\end{tabular}

Joseph D. Terwilliger, PhD

Department of Psychiatry, Columbia University

60 Haven Avenue -\#15C

New York, NY 10032 (USA)

E-Mailjdt3@columbia.edu 
rium - using analogies to subdivided populations with migration. We furthermore discuss how linkage disequilibrium, which has been generated by genetic drift in populations that have been of constant size over longer time periods, might be useful to find genes involved in common genetic diseases and discuss data from some populations of different demographic histories as well as some simulations to illustrate this prediction. In this context, we also demonstrate that for linkage disequilibrium mapping to work, one need not assume that a single ancestral mutation is responsible for a large proportion of disease cases in a present-day population, since genetic drift can create linkage disequilibrium around disease loci in the absence of such founder effects.

\section{The Evolution of Disease Loci and Surrounding Chromosomal Regions as 'Population Subdivision'}

Let us assume that at some arbitrary point in time, we can stratify a gene of interest into two classes of alleles, ' $\mathrm{D}$ ' $=$ the population of alleles which predispose to a phenotype of interest (e.g. disease), and ' + ' = the population of alleles at this locus which do not increase the probability of expressing said phenotype. Within the '+' class will be found the 'wildtype' form of this gene, and all of its polymorphisms which are phenotypically silent relative to our trait of interest, while the ' $D$ ' class contains all variants of the gene which increase the probability of expressing the phenotype. For example, in the BRCA1 gene there are hundreds of mutations with predisposing effects on breast cancer [1] - in this analysis those are considered as one ' $\mathrm{D}$ ' class of alleles, while in many diseases of the Finnish disease heritage $[2,3]$ there may be only one unique ancestral allele in this ' $\mathrm{D}$ ' class.
For marker loci located so closely to the disease locus that the recombination fraction between the disease and marker loci is effectively 0 , the population of chromosomes in the ' $D$ ' class will evolve independently of the population of chromosomes in the ' + ' class. This is true because without recombination, there is no opportunity for exchange of genetic material between the ' $D$ ' and ' + ' chromosomes. Thus, whereas the two populations of chromosomal regions carrying the ' $\mathrm{D}$ ' and ' + ' alleles evolve independently of one another (see fig. 1a), other unlinked parts of the genome evolve as one large population. Over time, this can lead to substantial differences in marker allele frequency distributions between the ' $D$ ' and ' + ' populations for markers in a neighborhood around the disease locus, even if there were no differences in the beginning. Our model demonstrates that linkage disequilibrium can exist even when the "basic assumption of linkage disequilibrium mapping - that a significant fraction of today's disease chromosomes derive from a common ancestor' [4] is violated, in that no linkage disequilibrium or single common ancestor need exist initially, for linkage disequilibrium to be generated over time.

The phenomenon of genetic drift generating linkage disequilibrium between neutral loci has been studied extensively, going back to seminal work of Hill and Robertson [5], and the analogy to subdivided populations of chromosomes provides an intuitive framework for studying the effects of drift in generating linkage disequilibrium, in the context of the more extensively studied problem of allele frequency variation between subdivided populations [6]. The dynamics of change in allele frequency due to genetic drift are mainly a function of population size. When the size of the ' $\mathrm{D}$ ' population is substantially smaller than that of the ' + ' population, as will typically be the case, genetic drift will cause a more 


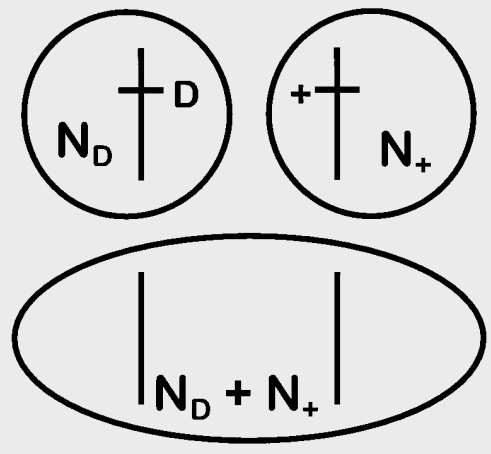

Fig. 1. a Absent recombination and mutation, the population of chromosomes with a ' $\mathrm{D}$ ' allele (size ' $\mathrm{N}_{\mathrm{D}}$ ') evolves independently of the population of chromosomes with a ' + ' allele ( size $\mathrm{N}_{+}$), while other chromosomes, on which the disease locus does not reside, evolve in concert as one large population of size $\mathrm{N}_{\mathrm{D}}+$ $\mathrm{N}_{+}$. b Effects of recombination fraction, $\theta$, in gene flow model of linkage disequilibrium. c Mutation at the disease locus as unidirectional gene flow from the '+' population to the ' $D$ ' population. Mutation rate from ' + ' to ' $\mathrm{D}$ ' is here considered to be equal to some small quantity $\mu$. d Population admixture/migration and its effects on linkage disequilibrium. In this case, we model the admixture from external population $B$ with frequency of allele ' $\mathrm{D}$ ' $=\mathrm{P}_{\mathrm{D} / \mathrm{B}}$, and total immigration of $\mathrm{N}_{\mathrm{B}}$ chromosomes into the total population $\mathrm{A}$ area. This would comprise $\mathrm{N}_{\mathrm{B}} \mathrm{P}_{\mathrm{D} / \mathrm{B}}$ new ' $\mathrm{D}$ ' chromosomes, and $\mathrm{N}_{\mathrm{B}}\left(1-\mathrm{P}_{\mathrm{D} / \mathrm{B}}\right)$ ' + ' chromosomes. Any differences in allele frequencies between ' $D$ ' and ' + ' chromosomes due to this admixture/migration would be due to differences between $P_{D}$ and $P_{D / B}$, and also differences in the distribution of allele frequencies at the marker locus between populations A and B. e 'Hitchhiking' effect when selection favors a given allele, leading to its increase in frequency in the population, due to varying causes, as described in fuller detail by Kimura [7, chap. 6]. In this diagram, some selective advantage is conferred to the $\mathrm{D}^{*}$ allele, and it increases in frequency, bringing along the 6 allele at the linked marker locus; this effect will alter the allele frequency distribution at the marker locus as shown with the 6 allele increasing through selection. Note that this selective effect could be an advantage of the $\mathrm{D}^{*}$ allele, or some other tightly linked allele on the same haplotype with D*.
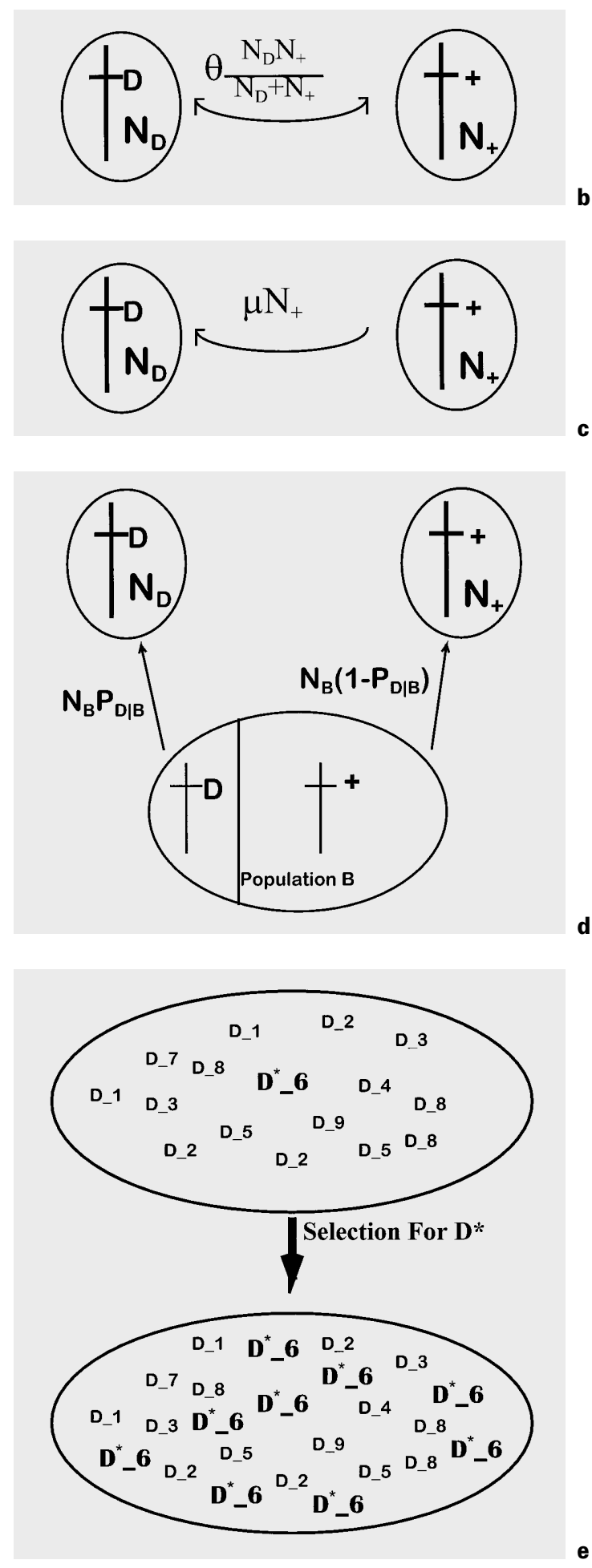

Terwilliger/Zöllner/Laan/Pääbo
140

Hum Hered 1998;48:138-154 
dramatic variance in allele frequencies from one generation to the next in the ' $\mathrm{D}$ ' population. In the simplest case of a diallelic marker locus, with frequency of $p_{n}$ in generation $n$, $\mathrm{E}\left(\mathrm{p}_{\mathrm{n}+1}\right)=\mathrm{p}_{\mathrm{n}}$, but $\operatorname{Var}\left(\mathrm{p}_{\mathrm{n}+1}\right)=\mathrm{p}_{\mathrm{n}}\left(1-\mathrm{p}_{\mathrm{n}}\right) / \mathrm{N}$ from simple binomial theory. Thus the larger the population size, the smaller the variance in allele frequency between generations, meaning that in large populations the frequency of an allele changes slower than in small ones. For this reason, drift will alter the allele frequency distributions at a faster rate in the smaller ' $D$ ' population than in the larger ' + ' population. The dynamics of this process for multiallelic loci are described in more complete mathematical detail elsewhere [7-9]. Generally, smaller populations acquire linkage disequilibrium faster than bigger ones.

It is important to point out that the linkage disequilibrium generated by genetic drift will take the form of strongly differing allele frequency distributions at marker loci in a neighborhood around a disease locus between ' $D$ ' and '+' chromosomes. However, this does not mean there will be a 'disease-associated haplotype'. In fact, there typically will not be such a single predominant haplotype - though there will be strong linkage disequilibrium between the disease locus and multiple markers. Certainly it is true that the distribution of haplotypes surrounding the disease locus will be different between ' $\mathrm{D}$ ' and ' + ' populations, but there will often be such haplotypic diversity within each class that haplotype-based analysis will often not be very sensitive. This results in a phenomenon more amenable to detection via multiple 2-point analysis [10, 11] than haplotype or shared segment analysis $[12,13]$.

In the case of a so-called 'founder effect', there is fixation of alleles in the ' $\mathrm{D}$ ' population for loci in a neighborhood around the ' $\mathrm{D}$ ' allele (i.e. a single disease-associated haplotype is created). If there were more than one, but still limited number of founders, then one might see a small number of haplotypes in this sample, the relative proportions of each varying over time due to genetic drift, with one or few likely becoming predominant over time as can be modeled using traditional genetic drift theory to predict the time to fixation of one allele (i.e. one of many 'D' alleles) conditional on population structure, etc. In this sense, the founder effect phenomenon can be generated in the population of ' $D$ ' alleles over time, as long as the absolute copy number of each ' $D$ ' allele remains sufficiently small. In this context, the insight of Cavalli-Sforza et al. [14] that a 'founder effect is clearly only an episode of drift' becomes apparent. In the early stages of expansion, while the absolute number of copies of each haplotype in the population remains small, drift does affect these haplotype frequency distributions - but as the population increases, and the absolute number of copies of each founder allele increases, the variance of its allele frequency in the next generation decreases, and genetic drift is arrested.

\section{Recombination as Symmetric and Bidirectional 'Gene Flow'}

To model the decay in linkage disequilibrium due to recombination we can consider recombination as bidirectional gene flow (or exchange) between the ' $D$ ' and ' + ' populations. The greater the recombination fraction between a marker locus and the disease gene, the greater the gene flow between ' $D$ ' and ' + ' populations. As is well known from population genetics, it does not take a substantial amount of gene flow to equilibrate the allele frequencies between populations. The equation for haplotype decay as proportional to $(1-\theta)^{n}$, where $\theta$ is the recombination fraction, is based on models of this gene flow (between 
'D' and ' + ' populations) and assumes that one starts with a 'founder effect' or fixation in the 'D' population for surrounding marker loci. If one does not have an equilibrium population, and there has been no fixation of single extended haplotypes in the ' $\mathrm{D}$ ' class (as is very often the case when disease alleles have combined frequency as large as 0.10), while recombination acts to equilibrate allele frequencies between the ' $\mathrm{D}$ ' and ' + ' populations, the genetic drift (acting faster in the ' $\mathrm{D}$ ' population than the ' + ' population because of differences in effective population size, $\mathrm{N}_{\mathrm{e}}$ ) works to generate new linkage disequilibrium over time. The relative impact of these two forces has been studied [15-17], and depends on the number of ' $\mathrm{D}$ ' chromosomes $\left(\mathrm{N}_{\mathrm{D}}\right)$ and the number of ' + ' chromosomes $\left(\mathrm{N}_{+}\right.$; see fig. $1 \mathrm{~b}$ ), such that the expected number of alleles exchanged between the two populations per generation is $\theta N_{D}\left(1-P_{D}\right)$, where $P_{D}$ is the frequency of the $\mathrm{D}$ chromosomes in the population: $P_{D}=N_{D} /\left(N_{D}+N_{+}\right)$. When this value is small, drift dominates over recombination, and when it is large, recombination decreases disequilibrium faster than drift can regenerate it. Note that when recombination acts alone, the ' $\mathrm{D}$ ' population in the next generation will on average have $\mathrm{N}_{\mathrm{D}}\left[1-\theta\left(1-\mathrm{P}_{\mathrm{D}}\right)\right]$ alleles derived from the previous generation's 'D' population, and $\mathrm{N}_{\mathrm{D}} \theta\left(1-\mathrm{P}_{\mathrm{D}}\right)$ alleles from the previous generation's ' + ' population, and thus the analogy to a population admixture model becomes clear.

\section{Mutation at Disease Locus as Unidirectional 'Gene Flow' between Populations}

The effects of mutations at the disease-predisposing locus will be to convert wild-type " + ' alleles into disease-predisposing alleles, which can be modeled as unidirectional gene flow from the ' + ' population to the ' $\mathrm{D}$ ' population (see fig. 1c). Such mutations will bring along a region of DNA around the disease locus and will therefore serve to alter the allele frequency distributions of linked marker loci. This effect will be more pronounced in the ' $\mathrm{D}$ ' population than in the ' + ' population, since the former population of chromosomes is generally smaller than the latter.

The mathematics of such migration models have been described, and can be applied to understand linkage disequilibrium dynamics. In fact, one can see that if the mutation rate from ' + ' to ' $D$ ' is $\mu$, and no back mutations occur, the number of alleles at a marker locus in the next generation's ' $D$ ' population derived from the previous generation's ' + ' population is expected to be $\mu N_{+}+\theta N_{D}\left(1-P_{D}\right)$, and the number of alleles derived from the previous generation's ' $D$ ' population is equal to $\mathrm{N}_{\mathrm{D}}\left[1-\theta\left(1-\mathrm{P}_{\mathrm{D}}\right)\right]$, as above. Thus, the population of ' $\mathrm{D}$ ' chromosomes is growing slowly due to mutations, since under most circumstances the rate of back mutation from ' $D$ ' to '+' can be considered to be infinitesimally small.

\section{Admixture Migration as Differential 'Migration' to Different Subpopulations}

It is well known that population admixture can create linkage disequilibrium [18]. The reason for this can also be described in the context of our model with ' $D$ ' and ' + ' populations as follows. If, for example, a group of individuals from a population $B$ that lack ' $D$ ' chromosomes migrate into a population $\mathrm{A}$, then all the incoming marker alleles go into the ' + ' class of chromosomes. The effect of this is to alter the distribution of nearby marker allele frequencies in the ' + ' chromosomes of population A, while leaving those in the ' $\mathrm{D}$ ' 
population unchanged. If the ' $\mathrm{D}$ ' class of alleles had frequency of 0.05 in population A, and 0.10 in population $B$, the admixture from population $B$ would affect the ' $D$ ' class in population A more than it would affect the ' + ' class, since the proportional amount of admixture would be greater, relative to $\mathrm{N}_{\mathrm{e}}$ (see fig. 1d). In general, we assume the ' $D$ ' class to be smaller than the ' + ' class, so the larger effects should typically be seen in ' $D$ '. Mathematically speaking, if the number of chromosomes per generation entering population $\mathrm{A}$ from population $B$ is $\mathrm{N}_{\mathrm{B}}$, and $\mathrm{P}_{\mathrm{D} / \mathrm{B}}$ is the frequency of the ' $D$ ' class of alleles in population $\mathrm{B}$, then $\mathrm{N}_{\mathrm{B}} \mathrm{P}_{\mathrm{D} / \mathrm{B}}$ chromosomes will migrate into the ' $\mathrm{D}$ ' class of alleles from population $\mathrm{B}$ to population $\mathrm{A}$ per generation, and $\mathrm{N}_{\mathrm{B}}\left(1-\mathrm{P}_{\mathrm{D} / \mathrm{B}}\right)$ chromosomes will migrate into the '+' class of alleles of population A per generation. Thus, under most circumstances, population admixture will tend to increase differences in the allele frequency distributions of linked marker loci between the ' $D$ ' and '+' populations.

\section{Selection Modeled as Change in Relative Size of Subpopulations and Hitchhiking Effect}

If selection against disease alleles occurs, the effect is to gradually reduce the size of the ' $\mathrm{D}$ ' population as well as the diversity at the disease locus and marker loci surrounding it. This will increase the rate of change of marker allele frequency distributions around the disease locus, leading to greater differences between ' $D$ ' and ' + ' populations. Selection can also act to increase linkage disequilibrium when the selection is in favor of some allele, or when there is balancing selection in a population to maintain some ' $D$ ' allele over time. Such selection will have its most striking effect when it is in favor of one ' $D$ ' allele over the others, such that allelic diversity in the ' $D$ ' class of alleles will decrease as one allele comes to predominate. This process can act in concert with random evolutionary forces to cause one allele to become fixed in the ' $D$ ' population. One striking effect can be to increase the size of the ' $\mathrm{D}$ ' population while decreasing its diversity - this effect alone can generate linkage disequilibrium over time, due to the 'hitchhiking effect' whereby the allele frequencies of existing alleles in the region around the disease allele whose frequency is rising in the population are rising along with it (see fig. 1e).

\section{Marker Locus Mutations - No Equilibrium State if $\mathrm{N}_{\mathrm{e}}$ Small}

Marker locus mutation rates have been thought of as an obstacle to doing linkage disequilibrium studies in human populations with microsatellites. It has long been known [8] that markers with more allelic diversity show higher amounts of linkage disequilibrium than less polymorphic markers, but it has been hypothesized that the higher mutation rates known to exist for microsatellite loci might preclude their usefulness in mapping studies - since mutation was thought of as a normalizing factor $[19,20]$. This makes intuitive sense if we are hypothesizing an equilibrium population in terms of the allele frequency distribution at a microsatellite locus. However, in practice, we are dealing with populations where at least the ' $D$ ' class is sufficiently small that equilibrium is not approached [21], at least not relative to mutation at the marker locus, such that any effects of mutation may act to increase disequilibrium by inducing asymmetric changes between the ' $\mathrm{D}$ ' and ' + populations (since marker mutation acts independently on each). Only in large populations, over the long term, may it lead to an approach 
to equilibrium and thus absence of linkage disequilibrium between the ' $D$ ' and ' + ' populations. It should be noted that in general, mutation rates for microsatellites are $<0.008$ [19], and when marker mutation rates on this order of magnitude (or smaller) are considered, the effects are negligible relative to genetic drift and recombination, and for the most part marker mutation can be safely disregarded as a factor in decay of drift-generated disequilibrium (see simulation results below).

\section{Inbreeding and Assortative Mating}

In general inbreeding and assortative mating will lead to a more rapid generation of linkage disequilibrium (due to its effect on decreasing the effective population size), and slower decay of such disequilibrium due to recombination - because in systems with inbreeding and in which there is assortative mating with respect to the genotype of the locus under study, the primary effect is that there will be too many individuals in the population who are homozygous at the disease locus, and too few heterozygotes [7, 8, 22]. In such cases, recombination will have less effect because it is increasingly likely that recombinations occur between two ' $\mathrm{D}$ ' chromosomes or between two ' + ' chromosomes. On the other hand, a tendency towards inbreeding avoidance may lead to recombination acting somewhat faster to decay the disequilibrium, though when the ' $\mathrm{D}$ ' allele frequency is $<0.20$, there are so few homozygotes expected that this effect is minimal. The effect on the gene flow between ' $D$ ' and ' + ' chromosomes can be quantified in terms of the inbreeding coefficient, $f$, such that instead of having $\theta \mathrm{N}_{\mathrm{D}} \mathrm{N}_{+} /\left(\mathrm{N}_{\mathrm{D}}+\mathrm{N}_{+}\right)$alleles at the marker locus flow between ' $\mathrm{D}$ ' and ' + ' populations each generation, one will have $\theta(1-f) \mathrm{N}_{\mathrm{D}} \mathrm{N}_{+} /\left(\mathrm{N}_{\mathrm{D}}+\mathrm{N}_{+}\right)[$see 23$]$.

\section{Constant Population Size versus Expanded Population Size}

Demographic history has dramatic impact on each of the aforementioned forces. In a population of constant size, new disequilibrium is continuously generated due to genetic drift [17]. In our example, random sampling of haplotypes causes a continual change in the marker allele frequencies in the ' $D$ ' and ' + ' populations, and also in the relative sizes of the ' $\mathrm{D}$ ' and ' + ' populations. In contrast, in a rapidly expanding population there will be little effect of genetic drift to generate new linkage disequilibrium (after the first few generations of expansion, when the absolute number of chromosomes is still small), since most alleles will be represented in each generation in roughly the same proportions as in the preceding generation. Consequently, the allelic composition of the population will be a snapshot of the conditions after the earliest stages of the expansion (which may itself represent the result of generations of earlier genetic drift). In such populations, however, there is a rapid decay in the amount of linkage disequilibrium around a given locus, because of the large number of opportunities for recombination and mutation, while in contrast to stable populations there is minimal genetic drift to generate new disequilibrium over time. Effectively, population expansion arrests genetic drift, and thus leads to populations in which new linkage disequilibrium does not develop, whereas recombination continues to decay existing linkage disequilibrium. A summary diagram of the forces affecting linkage disequilibrium in constant and expanded populations is outlined in figure 2 and table 1 ; the forces are described in terms of their relative influence on linkage disequilibrium.

We propose that the higher levels of linkage disequilibrium generated by genetic drift in constant populations may be usefully ex- 
Fig. 2. Summary of all the forces acting to generate and destroy linkage disequilibrium displayed in the context of our population subdivision model.

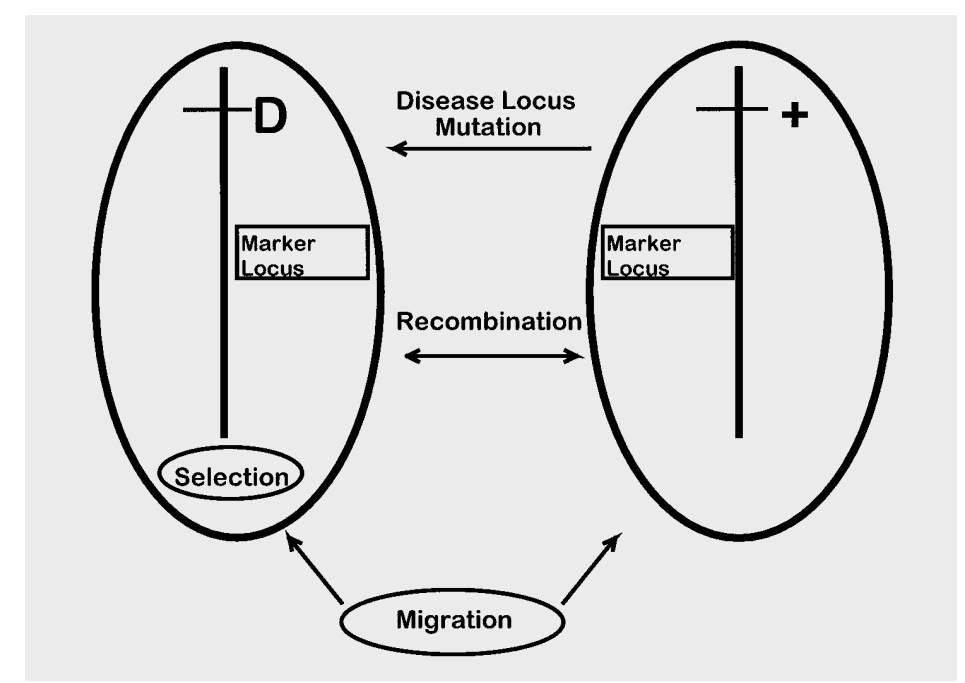

ploited as a tool to map genetic loci with reasonable phenotypic effects, including loci where disease alleles have been created by mutations far back in time and those at which multiple recurrent mutations have occurred over time. In this regard it is important to remember that what we mean by linkage disequilibrium is the existence of some difference in the allele frequency distribution at some marker locus conditional on whether ' $\mathrm{D}$ ' or ' + ' alleles are present at the disease locus. This will be the case for populations where no demographic expansion has taken place since genetic drift will there generate differences in marker allele frequency distributions in a neighborhood around the disease locus, whereas all unlinked parts of the genome evolve as one single population where consequently no differences in marker allele frequencies conditional on the disease trait are expected. In this approach, no single associated marker allele need be expected, and no single haplotype need be assumed. There will simply be higher levels of linkage disequilibrium around the disease locus, as described by Ohta and Kimura [15, 16] for selectively neu-

Drift Mapping in Population Isolates tral alleles, and by Franklin and Lewontin [24] and Felsenstein [25] in the presence of selection. At the time when the initial studies of drift-generated linkage disequilibrium were done [e.g. 5, 6, 15, 16, 24, 25], the major linkage disequilibrium mapping impetus was towards identifying rare alleles of strong effect, for which populations which had gone through a bottleneck followed by rapid expansion are ideal for detection of linkage disequilibrium. Today, however, the main focus of gene mappers is on identifying common alleles or allelic classes which may have smaller individual phenotypic effects. In this search, such expanded populations have not been very useful, and we propose that some of the solutions might lie rather in these stable populations which were ill-adapted to the earlier problem of identifying rare disease alleles.

In the last sections of this paper we investigate the possibilities for mapping through genetic drift in constant populations as compared with the common approach of using rapidly expanded populations to identify single haplotypes which appear due to a founder effect.

Hum Hered 1998:48:138-154 145 
Table 1. Effects of various phenomena on linkage disequilibrium mapping as a function of demographic history

\begin{tabular}{lll}
\hline Force & Population structure & \\
\cline { 2 - 3 } & constant size & exponential expansion \\
\hline Genetic drift & Increases & Negligible effect \\
Higher recombination fraction & Decreases & Decreases \\
Longer time in generations & Increases & Decreases \\
Large population size & Decreases & Decreases \\
Mutation (trait locus) & Minimal effect & Decreases \\
Mutation (marker locus) & Minimal effect & Minimal effect \\
Migration & Can increase & Slight increase possible \\
Shared segment methods? & No & Yes \\
Multiple 2-point works? & Yes & Yes \\
Protective alleles detected? & No & No \\
Founder effect? & No & Maybe \\
'D' allele frequency & Increases & Decreases \\
Pattern of disequilibrium & Random & Single haplotype \\
No initial disequilibrium & Generated over time & No later disequilibrium \\
\hline Decay rate in $\theta$ & Related to $\theta \frac{\mathrm{N}_{\mathrm{D}} \mathrm{N}_{+}}{\mathrm{N}_{\mathrm{D}}+\mathrm{N}_{+}}$ & Related to $(1-\theta)^{\mathrm{N}}$ \\
\hline
\end{tabular}

Where not clearly stated, the effects given are such when the phenomenon in the first column is increased. The effects listed under the population structure headings refer to the effects of the phenomenon on linkage disequilibrium.

\section{Saami and Finns}

Finns and Saami (formerly known as Lapps) are two populations that live in close geographic proximity in Fenno-Scandinavia. Whereas the Finns show signs of a rapid population expansion that probably took place within the last few thousand years, Saami do not present evidence of any such expansion. When seven microsatellite loci on the $\mathrm{X}$ chromosome were analyzed in random individuals in these two populations [26], it was found that 17 out of 21 pairwise comparisons of highly polymorphic microsatellite loci showed linkage disequilibrium at the $0.05 \mathrm{lev}$ el in a sample of Saami from northern Sweden, whereas only two pairs did so in the Finns. In fact, 14 of those pairs in Saami were significant at the 0.0001 level, while only 1 was in Finns; it should be noted that that single pair of very tightly linked loci which were strongly associated in Finns has been shown to be strongly associated ( $<<0.000001)$ in every population studied, irrespective of population structure or history [26].

Furthermore, several loci exhibiting linkage disequilibrium in the Saami were separated by more than $10 \mathrm{Mb}$. The same situation obtains in several other small populations that have been of constant size over long time according to analyses of DNA sequence variation, while many other large and small expanded populations behave like the Finns [Laan et al., unpubl. observations]. Thus, it seems that several nonexpanded human populations exhibit levels of drift-generated link- 
age disequilibrium that would potentially allow for the detection of disease loci by a genomic screen for linkage disequilibrium.

If one is applying haplotype analysis methods or searches for shared chromosomal segments, the high levels of marker to marker linkage disequilibrium may increase the rate of false positives [4, 27, 28]. Shared segment approaches are thus anticonservative because of the background linkage disequilibrium between markers - whether or not there is a disease allele in their vicinity. Furthermore, drift-generated linkage disequilibrium is not expected to present itself in the form of predominant shared segments or haplotypes. As a result, such approaches to gene mapping are not very powerful when linkage disequilibrium is present since it will not take this form. In contrast, single marker analysis should benefit from the marker-marker correlations - in that much less multiple testing correction is needed - much like the well-studied situation in linkage analysis [27, 29]. Multiple twopoint analysis $[10,11]$ is expected to be close to optimal for detecting this type of linkage disequilibrium, because the decay in the amount of disequilibrium between marker loci and disease locus is a function of the recombination fraction, and population size and structure. Methods based on the likelihood of multiple marker data as a function of this predicted decay in disequilibrium [see 11, 21] are under development.

In order to demonstrate empirically that the disequilibrium between linked markers does not increase the false-positive rate, the data of Laan and Pääbo [26] were subjected to a randomization analysis. In this analysis, we randomly assigned observed haplotypes of the seven loci in their study to be either cases or controls and then performed contingency table $\chi^{2}$ tests of linkage disequilibrium for each marker independently. From this, we determined the pointwise false-positive rate for each marker locus to show that there is no increased false-positive rate from this analysis. Then, we identified the most significant statistic over the seven markers analyzed, and looked at the false-positive rate over this region. For both samples, after 100,000 randomizations, the false-positive rates were consistent with what was expected for each of the marker loci in the sample, in a single marker locus analysis. However, when one identified the most significant of the seven loci, in Finns, the probability of this being significant at the 0.05 level was 0.27 , which is also the value one expects if the tests were independent at each locus (i.e. $1-\Pi\left[1-p_{i}\right]$ ), given the $\mathrm{p}_{\mathrm{i}}$ estimates from the randomizations. However, for the Saami, this probability was only 0.19 , where it was expected to be 0.265 if the tests were independent. For a $\mathrm{p}$ value of 0.01 , the corresponding false-positive rates were 0.042 in Finns, and 0.029 in Saami compared to expected (under independence) rates of 0.041 and 0.039 , respectively. This indicates that the presence of marker-marker disequilibrium, as predicted, leads to a decrease in the region-wide false-positive rates in the Saami, while in the rapidly expanded Finnish population, a Bonferroni correction is indicated because the tests of disequilibrium with even these tightly linked loci behave as independent tests.

\section{Simulated Population Data}

In order to simulate the extent to which the predictions outlined above can be expected to hold, simulations were performed for a given starting population size and frequency for the allelic class ' $D$ '. Disease locus genotypes were simulated assuming Hardy-Weinberg equilibrium, and marker locus genotypes (with phase) were simulated independently of the disease locus (i.e. no linkage disequilibrium is 

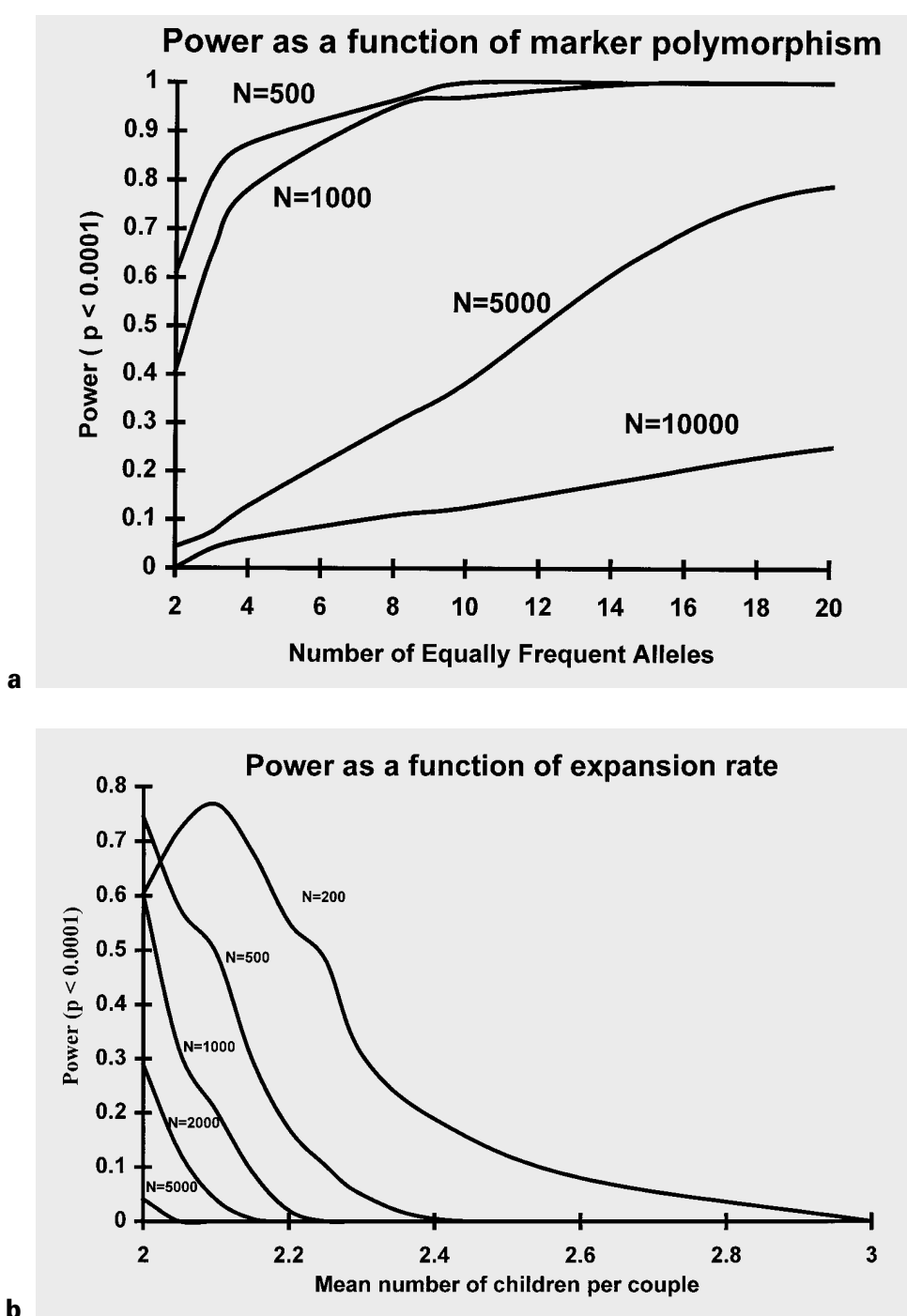

Fig. 3. Power calculations to demonstrate the effects of different phenomena on detectability of linkage disequilibrium between a marker locus and the disease locus genotypes. Note that for purposes of this figure, the simulated conditions were selected to demonstrate the effects of the various phenomena; thus the conditions do not represent the most powerful situations but rather situations for which the power varies as a function of the different parameters in an obvious manner. a Effects of marker heterozygosity - in this simulation, $\mathrm{P}\left({ }^{\prime} \mathrm{D}\right.$ ') $=0.20$, constant population sizes $(500,1,000$, 5,000 , and 10,000 founder individuals), and 30 generations since population founding with no initial disequilibrium. Marker heterozygosity is simulated by altering the number of equally frequent alleles ( $\mathrm{X}$ axis). When $\mathrm{P}\left({ }^{\prime} \mathrm{D}\right.$ ') is smaller, the powers are substantially higher; this value was selected to illustrate the effects of marker heterozygosity on linkage disequilibrium. b Effects of population expansion on linkage disequilibrium - in this simulation, $\mathrm{P}\left({ }^{\prime} \mathrm{D}\right.$ ') $=0.20$; the marker locus has 4 equally frequent alleles, and recombination fraction of 0.025 between disease and marker loci are assumed. When $\theta$ is smaller, the effect of expansion rate is attenuated somewhat, and when assumed to exist in the first generation). Mating pairs were selected randomly from the population, assuming one mate of opposite sex per individual, with the number of children per couple distributed according to a Poisson distribution with mean $\lambda$. When $\lambda=2$ the population size is roughly constant, and when $\lambda>2$ the population is exponentially expanding. Segregation of disease locus alleles to each child was random, with marker locus segregation being simulated conditional on disease locus transmission according to the recombination fraction, $\theta$. Mutations at the disease locus were simulated in one direction ' + ' $\rightarrow$ 'D' with small probability, back mutation assumed to be negligibly rare. At the marker locus mutations were assumed to follow a one-step model, wherein if a mutation occurs, the microsatellite allele size changes by one repeat unit up or down, with slight 
$\mathrm{P}\left({ }^{\prime} \mathrm{D}\right.$ ') is smaller the powers are higher in general. Initial population sizes $(\mathrm{N}=200,500,1,000$, 2,000, and 5,000) are shown; note that the populations are expanding exponentially, so the population from which the cases and controls were ascertained can be substantially larger than this number. c Effects of time and recombination fraction on linkage disequilibrium - in this simulation, $\mathrm{P}\left({ }^{\prime} \mathrm{D}\right.$ ') = 0.20 , and 4 equally frequent marker alleles are assumed - recombination fractions $(\theta=0,0.025,0.05$, and 0.15 ) and time in generations since the initial generation (where there was no disequilibrium) are varied to demonstrate their relative and combined effects. d Effects of recombination fraction on power to detect linkage disequilibrium. In this simulation, $\mathrm{P}\left({ }^{(} \mathrm{D}\right.$ ') $=0.20 ; 4$ equally frequent marker alleles, mutation rate $(+\rightarrow \mathrm{D})$ of 0.001 ; marker mutation rate of 0.008 , and population size $=1,000$. Power after 10,30 , and 50 generations is shown as a function of recombination fraction between disease and marker, $\theta$.
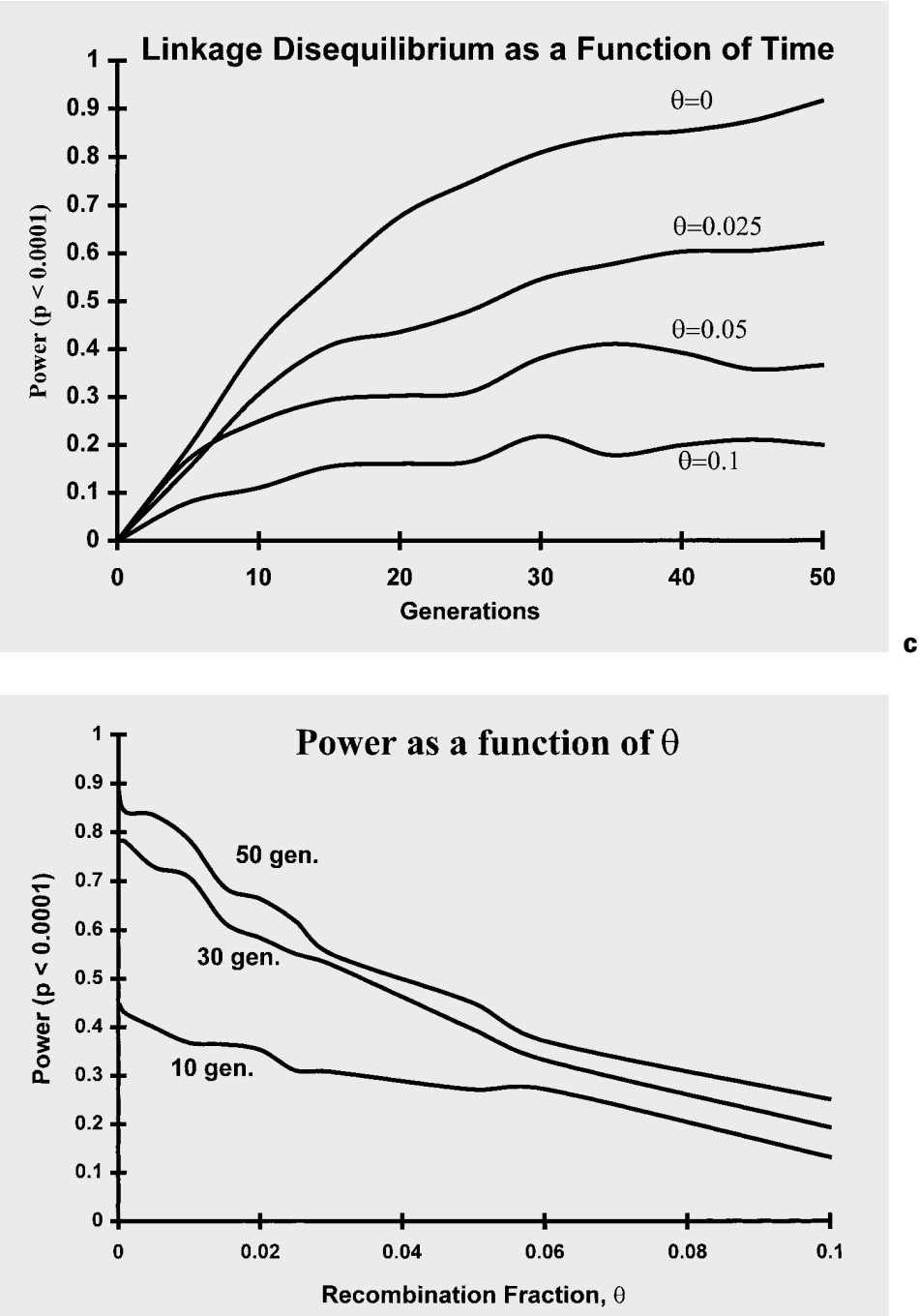

directional bias toward the center of the distribution. This process was repeated sequentially to simulate $\mathrm{N}$ generations.

To determine the power of the population to detect linkage disequilibrium, 100 chromosomes from the 'D' population and 100 from the ' + ' population were ascertained randomly and a contingency table $\chi^{2}$ test was performed; this was repeated 1,000 times to estimate the power to detect linkage disequilibri- um between alleles at the disease and marker loci for a given population structure after $\mathrm{N}$ generations. This power calculation was performed for each simulated replicate of the population history and structure. In each case, 100 such populations were simulated, with the average powers being presented in figure 3. To look at the effect of the mode of inheritance, trait phenotypes were simulated conditonal on genotypes at this locus accord- 
ing to some predetermined mode of inheritance, and again 50 cases and 50 controls (100 chromosomes per sample) were randomly ascertained from the population. Optionally parental controls can be used by this program according to the haplotype relative risk ascertainment procedure [see 30]. Selective neutrality was assumed for purposes of this study, but the software is written to allow for selection by censoring individuals from the next mating generation with probabilities conditional on disease locus genotype. This software is available from the authors by E-Mail request (to jdt3@columbia.edu) if the reader would like to simulate certain specific conditions, not given in this paper.

We first investigated the effects of marker heterozygosity on the power to detect linkage disequilibrium in constant populations of various sizes, as shown in figure 3a. In this analysis, we assumed the ' $D$ ' class of alleles to have frequency of $20 \%$, and absence of recombination between disease and marker loci. In each replicate, $n$ equally frequent alleles were simulated in populations of varying sizes, demonstrating that the more polymorphic the marker, the higher the chance to detect linkage disequilibrium, even when the marker locus mutation rate is assumed (as it was here) to be as high as 0.008 [19]. When the ' $D$ ' class of alleles is less frequent, the power can be substantially higher (data not shown) - the purpose of this analysis is to demonstrate the trend. It is clearly notable that smaller populations exhibit higher levels of disequilibrium than larger ones, also as predicted by the drift model. Larger populations can exhibit equally large levels of disequilibrium depending on the assumed distribution of marker allele frequencies and ' $\mathrm{D}$ ' allelic class frequency, consistent with empirical data [26].

To examine the effects of population expansion on the ability to detect linkage disequilibrium, we simulated populations with initial sizes of 200,500,1,000, 2,000, and 5,000 , with a mean number of offspring per couple ranging from 2 (constant population size) to 3 (rapid exponential growth of $150 \%$ per generation). The frequency of ' $D$ ' in the Nth generation was not specified or controlled, and the 'D' class alleles are sometimes completely lost in the smallest populations, or there were fewer than 100 ' $\mathrm{D}$ ' chromosomes in the population from which the data were ultimately ascertained - in those circumstances, the ' $\mathrm{D}$ ' sample is decreased as required, leading to the lower power in this sample, despite possibly higher levels of genetic drift. The results are shown in figure $3 \mathrm{~b}$. What one can see is that even small rates of population expansion prevent linkage disequilibrium from being generated in the 30 generations time-simulated in this analysis. In this example, a four-allele marker locus was simulated, with recombination fraction of 0.025 between marker and disease loci. The slight increase in power observed when moving from population size of 200 to population size of 500 (at no or very low expansion rates) has to do with the decreased probability of the ' $D$ ' class being eliminated due to drift in the larger population.

In figure 3c, we examine the effects of time and recombination fraction on the power to detect linkage disequilibrium - here in a constant population of starting size 1,000 , and a marker locus with 4 equally frequent alleles. As can be seen, the disequilibrium increases with time, to some stable 'equilibrium' between the effects of recombination fraction (decreasing disequilibrium) and genetic drift (increasing disequilibrium). The important factor here is that linkage disequilibrium created by genetic drift does not disappear with time, as opposed to linkage disequilibrium in rapidly expanded populations caused by a founder effect. As shown in the simulations in figure $3 \mathrm{~b}$, in expanded populations, 
the disequilibrium does disappear (or more accurately fails to appear) as time increases. In light of these results we propose that small populations of constant size might be of more utility in gene mapping of common ' $D$ ' classes of alleles. To address the issue of how quickly the disequilibrium decays with respect to the recombination fraction, figure $3 \mathrm{~d}$ shows this relationship in terms of power to detect the disequilibrium assuming a population of constant size 1,000, a 4-allele marker, and P('D') $=0.20$ as above. As one can see, the power does drop substantially as one goes to increasing genetic distances, but consistent with the results in figure $3 \mathrm{c}$, the power is still fairly large even at a genetic distance of $\theta=0.10$.

To analyze the observation that in isolated populations of constant size there is an abject absence of rare recessive diseases [31], in contrast to the diseases of the Finnish disease heritage $[2,3]$ (which remain in large numbers due to rapid population expansion), ' $\mathrm{D}$ ' allele frequencies of 0.01 and 0.001 were simulated, in which case fixation of the ' + ' allele occurs rapidly in the constant populations, while in the expanded populations there was much higher probability of it being maintained through the expansion (data not shown). This observation was consistent with empirical evidence. Similarly in those situations where a single founder chromosome was maintained, the predicted single haplotype was also preserved over a long distance around the disease locus consistent with predictions and empirical evidence.

When one is hoping to detect allelic associations with a complex phenotype, one will obviously need larger sample sizes than to detect linkage disequilibrium between markers and disease locus genotypes [32-35]. Just how much lower the power will be is a function of the genotype-phenotype relationship, the distribution of alleles at other disease-predisposing loci in a given population, and other etiological covariates (be they genetic, environmental, or cultural). In small populations of stable demographic history, the chance for fixation of alleles at additional loci involved in the etiology of disease is high $[7,8]$, potentially increasing or decreasing the power (due to the potentially increased amount of genetic homogeneity in the population and correspondingly lower levels of genotypic and phenotypic variability).

\section{Complex Disease Genetics and Drift Mapping}

There has been a great deal of effort invested to try and identify genetic factors which have some predisposing influence on common phenotypes. To date only linkage analyses on large pedigrees [e.g. 36-39] and candidate gene analyses [e.g. 40-42] have been successful in gene identification. To this end, it has been proposed that linkage disequilibrium methods might provide a solution [32]. However, as these results indicate (see fig. 3b) such studies may be doomed to failure in expanded populations. Most linkage disequilibrium mapping studies have looked at populations which have undergone a bottleneck followed by rapid expansion to try and identify unique disease-associated haplotypes. This protocol can be successful only in diseases where the ' $\mathrm{D}$ ' allele is rare (relative to $\mathrm{N}_{\mathrm{e}}$ ) and its effect on the phenotype is large [e.g. 12, 13, 43, 44], and/or selective pressure in the past may have led to an increase in the frequency of a specific ' $D$ ' allele at some point in history [44, 45]. It is proposed that small populations of constant size (including those with recent admixture) might hold some of the solutions to this problem [see 46-49] provided that sufficient epidemiological information can be made available [see 50-57]. 
It has been questioned [4] whether '... the allelic complexity of common diseases [will] turn out to be sufficiently low for LD mapping to work, even in young isolated populations'. If one considers this model for drift-generated linkage disequilibrium, it may turn out that allelic complexity is not such a significant impediment, but that young isolated populations may be far from ideal when disease alleles are common. Perhaps it is in the older constant-sized populations where some of the solutions may be found.

\section{Conclusions}

In summary of the model, let us refer back to figure 2 , in which the major forces affecting the dynamics of linkage disequilibrium are illustrated, and table 1 in which the effects of the various phenomena are delineated. It is clear that for mapping of rare diseases of strong effect, populations which have undergone a bottleneck followed by rapid expansion may be optimal, as evidenced by the successful mapping studies of the Finnish disease heritage [2, 58-60]. This same result may apply if selective pressure may have caused a disease allele to increase in frequency at some point in the past due to a hitchhiking effect. However, for disease alleles which are common relative to the population size, and for which selective neutrality is assumed (for most common oligogenic diseases - typically of late age of onset - selection can largely be ignored), there may be no detectable linkage disequilibrium in these rapidly expanded populations.

In contrast, populations of small constant size generate new disequilibrium faster than recombination can make it disappear, such that in large populations the disequilibrium is only generated over small regions, while in small populations it can extend over very large regions. This disequilibrium, while not presenting a 'disease-associated' haplotype, can be detected in case control studies using two-point or multiple pairwise approaches. To this end, for mapping of disease loci whose ' $\mathrm{D}$ ' class of alleles is large relative to $\mathrm{N}_{\mathrm{e}}$, small constant populations may provide the best solution. This may be so, not only because of the high amount of linkage disequilibrium in such populations, but also because for oligogenic traits, there is likely to be a smaller number of additonal predisposing genes segregating in those populations (other disease alleles at other loci may be fixed or lost to drift with higher probability than in expanded populations), as well as higher levels of cultural and environmental homogeneity. When combined with recent colonial admixture, there may be a plethora of allelic associations waiting to be discovered in the right resource. Anthropological geneticists have already used extremely isolated populations in linkage mapping studies [e.g. 61, 62], and it is proposed that further collaboration between population geneticists and gene mappers might be critical to making a success of common disease gene mapping by linkage disequilibrium.

\section{Acknowledgments}

We thank the following for financial support: A Hitchings-Elion Fellowship from the Burroughs-Wellcome Fund (to J.T.), a fellowship from the Alexandervon-Humboldt-Stiftung (to M.L.), and the Deutsche Forschungsgemeinschaft ( $\mathrm{Pa}$ 452/3-1; Ha1628/2-2). Helpful comments on an earlier version of this manuscript from Harald Göring, Dan Rabinowitz, Jürg Ott, and an anonymous reviewer are gratefully acknowledged as are useful discussions with Jim Knowles and Michael Crawford.
152

Hum Hered 1998;48:138-154

Terwilliger/Zöllner/Laan/Pääbo 


\section{References}

1 Grade K, Jandrig B, Scherneck S: BRCA1 mutation update and analysis. J Cancer Res Clin Oncol 1996; 122:702-706.

2 Norio R: Suomalaisen tautiperinnön tulevaisuus. Duodecim 1994; 110:640-643.

3 Norio R, Nevanlina HR, Perheentupa J: Hereditary diseases in Finland: Rare flora in rare soil. Ann Clin Res 1973;5:109-141.

4 Kruglyak L: What is significant in whole-genome linkage disequilibrium studies? Am J Hum Genet 1997; 61:810-812.

5 Hill WG, Robertson A: Linkage disequilibrium in finite populations. Theor Appl Genet 1968;38:226231.

6 Ohta T: Linkage disequilibrium due to random genetic drift in finite subdivided populations. Proc Natl Acad Sci USA 1982;79:1940-1944.

7 Kimura M: The Neutral Theory of Molecular Evolution. Cambridge, Cambridge University Press, 1983.

8 Wright S: Evolution and the Genetics of Populations, vol 2: The Theory of Gene Frequencies. Chicago, University of Chicago Press, 1969.

9 Cavalli-Sforza LL, Bodmer WF: The Genetics of Human Populations. San Francisco, Freeman 1971.

10 Morton NE, Andrews V: MAP, an expert system for multiple pairwise linkage analysis. Ann Hum Genet 1989;53:263-269.

11 Terwilliger JD: A powerful likelihood method for the analysis of linkage disequilibrium between trait loci and one or more polymorphic marker loci. Am J Hum Genet 1995;56: 777-787.

12 Houwen RHJ, Baharloo S, Blankenship K, Raeymaekers P, Juyn J, Sandkuyl LA, Freimer NB: Genome screening by searching for shared segments: Mapping a gene for benign recurrent intrahepatic cholestasis. Nat Genet 1994;8:380-386.

13 Nikali K, Suomalainen A, Terwilliger J, Koskinen $\mathrm{T}$, Weissenbach $\mathbf{J}$ Peltonen L: Random search for shared chromosomal regions in four affected individuals: The assignment of a new hereditary ataxia locus. Am J Hum Genet 1995;56: 1088-1095.
14 Cavalli-Sforza LL, Menozzi P, Piazza A: The History and Geography of Human Genes. Princeton, Princeton University Press, 1994.

15 Ohta T, Kimura M: Linkage disequilibrium due to random genetic drift. Genet Res 1969;13:47-55.

16 Ohta T, Kimura M: Linkage disequilibrium at steady state determined by random genetic drift and recurrent mutation. Genetics 1969; 63:229-238.

17 Slatkin M: Linkage disequilibrium in growing and stable populations. Genetics 1994;137:331-336.

18 Chakraborty R, Weiss K: Admixture as a tool for finding linked genes and detecting that difference from allelic association between loci. Proc Natl Acad Sci USA 1988;85:91199123.

19 Weber JL, Wong C: Mutation of short tandem repeats. Hum Mol Genet 1993;2:1123-1128.

20 Todd JA: Panning for gold: Genome-wide scanning for linkage in type I diabetes. Hum Mol Genet 1992;1:663-666.

21 Kaplan NL, Hill WG, Weir BS: Likelihood methods for locating disease genes in nonequilibrium populations. Am J Hum Genet 1995;56: 18-32.

22 Maynard Smith J: Evolutionary Genetics. Oxford, Oxford University Press, 1989.

23 Hartl DL: A Primer of Population Genetics. Sunderland, Sinauer, 1987.

24 Franklin I, Lewontin RC: Is the gene the unit of selection? Genetics 1970 65:707-734.

25 Felsenstein J: Uncorrelated genetic drift of gene frequencies and linkage disequilibrium in some models of linkage overdominant polymorphisms. Genet Res Camb 1974;24: 281-294.

26 Laan M, Pääbo S: Demographic history and linkage disequilibrium in human populations. Nat Genet 1997; 17:435-438.
27 Terwilliger JD, Shannon WD, Lathrop GM, Nolan JP, Goldin LR, Chase GA, Weeks DE: True and false peaks in genome-wide scans: Applications of length-biased sampling to linkage mapping. Am J Hum Genet 1997;61:430-438.

28 Terwilliger JD: Mapping genes predisposing to complex traits in extreme population isolates. CSC News 1997;02/97:23-26.

29 Lander ES, Kruglyak L: Genetic dissection of complex traits: Guidelines for interpreting and reporting linkage results. Nat Genet 1995;11:241247.

30 Terwilliger JD, Ott J: A haplotypebased haplotype relative risk statistic. Hum Hered 1992;42:337-346.

31 Milan FA: The Human Biology of Circumpolar Populations. Cambridge, Cambridge University Press, 1980.

32 Risch N, Merikangas K: The future of genetic studies of complex human diseases. Science 1996;273:15161517.

33 Weiss KM: Genetic Variation and Human Disease. Cambridge, Cambridge University Press, 1995.

34 Weiss KM: Is there a paradigm shift in genetics? Lessons from the study of human diseases. Mol Phylogenet Evol 1996;5:259-265.

35 Terwilliger JD: Review of 'Genetic Variation and Human Disease' by Weiss KM. Am J Hum Genet 1997; 60:1565-1566.

36 Goldgar DE, Fields P, Lewis CM, Tran TD, Cannon-Albright LA, Ward JH, Swensen J, Skolnick MH: A large kindred with 17q-linked breast and ovarian cancer: Genetic, phenotypic, and genealogical analysis. J Natl Cancer Inst 1994;86:200 209.

37 Trembath RC, Clough RL, Rosbotham JL, Jones AB, Camp RDR, Frodsham A, Browne J, Barber R, Terwilliger JD, Lathrop GM, Barker JNWN: Identification of a major susceptibility locus on chromosome $6 \mathrm{p}$ and evidence for further disease loci revealed by a two stage genomewide search in psoriasis. Hum Mol Genet 1997;6:813-820. 
38 Pericak-Vance MA, Bebout JL, Gaskell PC Jr, Yamaoka LH, Hung WY, Alberts MJ, Walker AP, Bartlett RJ, Haynes CA, Welsh KA, et al: Linkage studies in familial Alzheimer disease: Evidence for chromosome 19 linkage. Am J Hum Genet 1991; 48:1034-1050.

39 Tienari PJ, Terwilliger JD, Ott J, Palo J, Peltonen L: Two-locus linkage analysis in multiple sclerosis. Genomics 1994;19:320-325.

40 Spielman RS, McGinnis RE, Ewens WJ: Transmission test for linkage disequilibrium: The insulin gene region and insulin-dependent diabetes mellitus (IDDM). Am J Hum Genet 1993;52:506-516.

41 McKenzie CA, Julier C, Forrester T, McFarlane-Anderson N, Keavney B, Lathrop GM, Ratcliffe PJ, Farrall $\mathrm{M}$ : Segregation and linkage analysis of serum angiotensin I-converting enzyme levels: Evidence for two quantitative trait loci. Am J Hum Genet 1996;57:1426-1435.

42 Risch N: Assessing the role of HLAlinked and unlinked determinants of disease. Am J Hum Genet 1987; 40: $1-14$.

43 deVries HG, van der Meulen MA Rozen R, Halley DJ, Scheffer H, ten Kate LP, Buys CH, te Meerman GJ: Haplotype identity between individuals who share a CFTR mutation allele 'identical by descent': Demonstration of the usefulness of the haplotype-sharing concept for gene mapping in real populations. Hum Genet 1996;98:304-309.

44 Ajioka RS, Jorde LB, Gruen JR, Yu P, Dimitrova D, Barrow J, Radisky E, Edwards CQ, Griffen LM, Kushner JP: Haplotype analysis of hemochromatosis: Evaluation of different linkage-disequilibrium approaches and evolution of disease chromosomes. Am J Hum Genet 1997;60:1439-1447.
45 Escamilla MA, Spesny M, Reus VI, Gallegos A, Meza L, Molina J, Sandkuijl LA, Fournier E, Leon PE, Smith LB, Freimer NB: Use of linkage disequilibrium approaches to map genes for bipolar disorder in the Costa Rican population. Am J Med Genet 1996;67:244-253

46 Harvald B: Breakup of an isolate. Arctic Med Res 1988;47:41-42.

47 Harvald B: The genetic epidemiology of Greenland. Arctic Med Res 1989;48:171-174.

48 Nordic Council for Arctic Medical Research: Symposium on genetic diseases in the sparsely populated areas of the Nordic countries. Oulu, Report 24, 1979.

49 Terwilliger JD: Genetic epidemiology and circumpolar population isolates - The 'Finland' of complex disease? Abstract to Securing Northern Futures. Canadian Circumpolar Institute, Edmonton 1997.

50 Andersen S: Greenland medical bibliography. Arctic Med Res 1981; 29:1-137.

51 Curtis T, Bjerregaard P: Health Research in Greenland - A Catalogue of Projects. Danish Institute of Clinical Epidemiology, Copenhagen 1995.

52 Fortuine R: The health of the Inuit of North America: A bibliography from the earliest times through 1990. Arctic Med Res 1993; 52(suppl 8):1-353.

53 Kaznacheev VP, Kulikov VJ, Soli E, Leppäluoto J, Stenbäck F: Bibliography on arctic medical research in the USSR Arctic Med Res 1985;39:1143.
54 Kromann N, Green A: Epidemiological studies in the Upernavik District, Greenland: Incidence of some chronic diseases 1950-74. Acta Med Scand 1980;208:401-406.

55 Shephard RJ, Rode A: The Health Consequences of 'Modernization'. Cambridge, Cambridge University Press, 1996.

56 Nielsen NH: Cancer incidence in Greenland. Arctic Med Res 1986; 43:1-168.

57 Young TK, Schraer CD, Shubnikoff EV, Szathmary JE, Nikitin YP: Prevalence of diagnosed diabetes in circumpolar indigenous populations. Int J Epidemiol 1992;21:730 736 .

58 Nevanlinna HR: The Finnish population structure. A genetic and genealogical study. Hereditas 1972;71: 195-236.

59 de la Chapelle A, Hästbacka J, Lehesjöki AE, Sulisalo T, Kere J, Tahvanainen E, Sistonen P: Kytkentä ja kytkentäepatasapaino suomalaisessa tautiperinnössä. Duodecim 1994; 110:654-664.

60 Peltonen L, Pekkarinen P, Aaltonen $\mathrm{J}$ : Messages from an isolate: Lessons from the Finnish gene pool. Biol Chem Hoppe-Seyler 1995;376:697704.

61 Eiberg H, Nielsen IM: Linkage studies of cholestasis familiaris groenlandica/Hyler-like disease with polymorphic protein and blood group markers. Hum Hered 1993;43:250 256.

62 Norman RA, Thompson DB, Foroud T, Garvey WT, Bennett PH, Bogardus C, Ravussin E: Genomewide search for genes influencing percent body fat in Pima Indians: Suggestive linkage at chromosome 11q21-q22. Pima Diabetes Gene Group. Am J Hum Genet 1997;60: 166-173. 